\title{
BreastCore
}

\section{HER2-Orientated Therapy in Early and Metastatic Breast Cancer}

\author{
Severine Iborra Elmar Stickeler \\ Department of Gynecology and Obstetrics, University Medical Center Aachen, Aachen, Germany
}

\author{
Keywords \\ Targeted therapy · HER2 - Early breast cancer . \\ Metastatic breast cancer
}

\section{Summary}

Due to the enhanced understanding of molecular oncology and signaling pathways in breast cancer (BC), therapy management has undergone a major transformation, especially with the emergence of treatment tailored to individual disease characteristics. In the case of HER2positive early or metastatic $\mathrm{BC}$, targeted therapies are well established and remain a major focus of ongoing research. The introduction of anti-HER2 biologicals such as trastuzumab, pertuzumab, and T-DM1 has made targeted and personalized treatment possible and has clearly improved disease-free and overall survival in patients with HER2-positive BC. Moreover, neoadjuvant chemotherapy represents a well-established and often favored option for patients with operable BC and a clear indication for postoperative chemotherapy (such as HER2-positive BC). Other trials are trying to identify additional surrogate markers for therapy response and clinical outcome in the neoadjuvant setting and that way open up new perspectives with a possible de-escalation of classical treatment in favor of targeted therapy.

(c) 2016 S. Karger GmbH, Freiburg

\section{Introduction}

Breast cancer (BC) remains the most common cancer in women worldwide. The systemic therapeutic management of $\mathrm{BC}$ has undergone a significant transformation during the past decade. The progress in molecular oncology and the enhanced understanding of signaling pathways have led to the emergence of targeted therapy and have made treatments tailored to individual disease characteristics possible.

The human epidermal growth factor receptor 2 (HER2) has been shown to be overexpressed in $20-30 \%$ of human $\mathrm{BC}$ cell lines [1]. Overexpression of HER2 in BC seems to confer a more aggressive phenotype and, historically, was associated with a poor prognosis with higher risk of recurrence, lower disease-free survival (DFS) and overall survival (OS) rates, and greater resistance to treatment [2-5]. HER2 belongs to the human epidermal growth factor receptor family consisting of 4 transmembrane tyrosine kinase receptors, namely HER1 (ErbB1), HER2 (Neu, ErbB2), HER3 (ErbB3), and HER4 (ErbB4) $[2,6,7]$. All of these receptors have a similar structure. They normally regulate cell growth and survival as well as adhesion, migration, differentiation, and other cellular responses. Ligand binding to ErbB receptors results in dimerization of 2 receptors (homodimerization if 2 identical receptors, heterodimerization if not) and activates signaling cascades. Specifically, HER2 has no known ligand and if amplified can activate other HER family members in the absence of ligands [8]. This procedure activates signaling cascades that induce cell proliferation via the Ras/MAPK (mitogen-activated protein kinases) pathway and inhibit cell death via the PI3K/Akt/mTOR (phosphatidylinositol 3-kinase/protein kinase $\mathrm{B} /$ mammalian target of rapamycin) pathway [9].

\section{HER2-Targeted Drugs}

\section{Trastuzumab}

Trastuzumab, a recombinant monoclonal antibody targeting the extracellular subdomain IV of HER2, was the first biological drug approved for the treatment of HER2-positive BC and revolutionized the management of both early and advanced BC. Trastuzumab acts via different mechanisms to inhibit cell growth: prevention of HER2 dimerization, downregulation of the HER2 receptor by endo-

\section{KARGER}

(c) 2016 S. Karger GmbH, Freiburg

Fax +497614520714 
Table 1. Overview of the different HER2orientated therapies

\begin{tabular}{|c|c|c|c|c|}
\hline Substance & $\begin{array}{l}\text { Mode } \\
\text { of action }\end{array}$ & Side effects & Indication & Regimen \\
\hline \multirow[t]{3}{*}{$\begin{array}{l}\text { Trastuzumab } \\
\text { Herceptin }^{\circledR a}\end{array}$} & \multirow[t]{3}{*}{$\begin{array}{l}\text { recombinant } \\
\text { monoclonal } \\
\text { antibody }\end{array}$} & \multirow[t]{3}{*}{$\begin{array}{l}\text { reversible cadiotoxicity, } \\
\text { flu-like symptoms, } \\
\text { myelosuppression, } \\
\text { nausea and emesis, } \\
\text { allergy }\end{array}$} & NACT & $\begin{array}{l}\text { in combination/concurrent with: } \\
\text { a) } \mathrm{P} \text { or } \mathrm{D} \text { after } 4 \times \mathrm{EC}(\mathrm{AGO}++) \\
\text { b) } \mathrm{P} \text { or } \mathrm{D}+\text { pertuzumab after } 4 \times \mathrm{EC}(\mathrm{AGO}+)\end{array}$ \\
\hline & & & $\begin{array}{l}\text { adjuvant } \\
\text { therapy }\end{array}$ & $\begin{array}{l}\text { a) } \mathrm{P} \text { or } \mathrm{D} \text { after } 4 \times \mathrm{EC}(\mathrm{AGO}++) \\
\text { b) } 12 \times \mathrm{P} \text { without } \mathrm{EC} \text { if tumor }<3 \mathrm{~cm} \text { and } \\
\text { pN0 }(\mathrm{AGO}+) \\
\text { c) } \mathrm{D} \text { and } \mathrm{C}(\mathrm{AGO}+)\end{array}$ \\
\hline & & & $\mathrm{MBC}$ & $\begin{array}{l}\text { a) } \mathrm{D}+\text { pertuzumab }(\mathrm{AGO}++) \\
\text { b) } \mathrm{P}+\text { pertuzumab }(\mathrm{AGO}+) \\
\text { c) 1st-line chemotherapy } \\
\text { d) lapatinib as 2nd-line therapy }(\mathrm{AGO}+) \\
\text { e) AI as 2nd-line therapy }(\mathrm{AGO}+)\end{array}$ \\
\hline \multirow[t]{2}{*}{$\begin{array}{l}\text { Pertuzumab } \\
\text { Perjeta }^{\circledR a}\end{array}$} & \multirow[t]{2}{*}{$\begin{array}{l}\text { recombinant } \\
\text { monoclonal } \\
\text { antibody }\end{array}$} & \multirow[t]{2}{*}{$\begin{array}{l}\text { neutropenia, diarrhea, } \\
\text { nausea, alopecia, skin rash, } \\
\text { peripheral neuropathy, } \\
\text { cardiac dysfunction or failure }\end{array}$} & NACT & $\begin{array}{l}\text { in combination/concurrent with: } \\
\mathrm{P} \text { or } \mathrm{D}+\text { trastuzumab after } 4 \times \mathrm{EC}(\mathrm{AGO}+)\end{array}$ \\
\hline & & & $\mathrm{MBC}$ & $\begin{array}{l}\text { a) } \mathrm{D}+\text { trastuzumab }(\mathrm{AGO}++) \\
\text { b) } \mathrm{P}+\text { trastuzumab }(\mathrm{AGO}+)\end{array}$ \\
\hline $\begin{array}{l}\text { T-DM1 } \\
\text { Kadcyla }^{\circledR a}\end{array}$ & $\begin{array}{l}\text { antibody-drug } \\
\text { conjugate }\end{array}$ & $\begin{array}{l}\text { fatigue, anemia, nausea, } \\
\text { hypokalemia, } \\
\text { thrombocytopenia, increased } \\
\text { transaminases }\end{array}$ & $\mathrm{MBC}$ & $\begin{array}{l}\text { monotherapy: } \\
\text {-in the 1st line if recurrence within } 6 \text { months } \\
\text { after therapy with taxane + trastuzumab } \\
(\mathrm{AGO}+) \\
\text {-in the } 2 \text { nd-line }(\mathrm{AGO}++) \\
\text {-in further lines }(\mathrm{AGO}++)\end{array}$ \\
\hline $\begin{array}{l}\text { Lapatinib } \\
\text { Tyverb }^{\circledR b}\end{array}$ & $\begin{array}{l}\text { dual tyrosine } \\
\text { kinase } \\
\text { inhibitor }\end{array}$ & $\begin{array}{l}\text { skin rash (exanthema), } \\
\text { palmar-plantar erythro- } \\
\text { dysesthesia, diarrhea, } \\
\text { nausea, hepatotoxicity }\end{array}$ & $\mathrm{MBC}$ & $\begin{array}{l}\text { in combination/concurrent with: } \\
\text {-trastuzumab as 2nd- and further line therapy } \\
(\mathrm{AGO}+) \\
-\mathrm{AI} \text { as } 2 \text { nd-line therapy }(\mathrm{AGO}+) \\
\text {-capecitabin as } 2 \text { nd- and further line } \\
\text { therapy }(\mathrm{AGO}+)\end{array}$ \\
\hline
\end{tabular}

aRoche Pharma AG, Grenzach-Whylen, Germany.

${ }^{b}$ Novartis Pharma GmbH, Nuremberg, Germany.

$\mathrm{NACT}=$ Neoadjuvant therapy; $\mathrm{MBC}=$ metastatic breast cancer; $\mathrm{D}=$ docetaxel, $\mathrm{P}=$ paclitaxel $\mathrm{C}=$ carboplatin; $\mathrm{EC}=$ epirubicin + cyclophosphamide; $\mathrm{AI}=$ aromatase inhibitor. cytic destruction of the receptor, induction of cell cycle arrest, induction of antibody-dependent cellular cytotoxicity, inhibition of DNA repair, decreased angiogenesis, and impairment of constitutive HER2 extracellular domain cleavage $[10,11]$. The major described side effect of trastuzumab is a reversible cardiotoxicity. Otherwise, when used alone, myelosuppression, nausea, and emesis occur in rare cases. An acute hypersensitivity-like reaction has been described in less than $10 \%$ of patients. With the significant advances in the understanding of the molecular mechanism of trastuzumab activity as well as resistance since its first approval in 1998, current research focusses on the development of new drugs to improve anti-HER2 activity and overcome resistance (table 1).

\section{Pertuzumab}

Pertuzumab is a recombinant monoclonal antibody that binds to another epitope in the extracellular domain of HER2 (subdomain II). Pertuzumab acts as a dimerization inhibitor between
HER2 and the other HER family receptors by preventing the ligand-induced HER2 heterodimer, and that way provides a complementary mechanism of action to that of trastuzumab which inhibits the signaling pathway without affecting dimerization. Moreover, pertuzumab as an antibody activates the immune system, especially the natural killer cells, and therefore shows an antitumoral activity through antibody-dependent cellular cytotoxicity $[12,13]$. Pertuzumab and trastuzumab synergistically block the survival of HER2-overexpressing breast cells [14]. Known toxicities of treatment with pertuzumab are neutropenia, diarrhea, nausea, alopecia, skin rash, and peripheral neuropathy. Like trastuzumab, cardiac dysfunction or heart failure have been described (table 1).

\section{Ado-Trastuzumab Emtansine (T-DM1)}

T-DM1 is an antibody-drug conjugate (ADC) consisting of the monoclonal antibody trastuzumab as a linker and the cytotoxic agent emtansine (DM1), derivative of maytansine, an antimitotic 
drug. This trastuzumab conjugate simply uses HER2 as an address for the targeted delivery of a potent cytotoxic agent, emtansine, into the cell. The collateral damage to normal cells and related systemic toxicity is thus limited using treatment with ADC [15-17]. Described side effects are fatigue, anemia, nausea, hypokalemia, thrombocytopenia, and increased transaminases $[15,18]$ (table 1).

\section{Lapatinib}

Lapatinib is a dual tyrosine kinase inhibitor as it reversibly inhibits ErbB1 and ErbB2 tyrosine kinases. Phosphorylation and activation of cell proliferation effectors such as MAPK and Akt are subsequently blocked in ErbB1- and ErbB2-expressing tumor cell lines [19-22]. Known side effects are skin rashes (exanthema), palmar-plantar erythrodysesthesia, diarrhea, nausea, and hepatotoxicity (table 1).

\section{HER2-Targeted Therapy in Early Breast Cancer}

\section{Neoadjuvant Therapy (NACT)}

Currently, NACT represents a well-established as well as often favored option for patients with operable BC. Survival rates after NACT are comparable with those after adjuvant therapy $[23,24]$ since NACT contributes to surgical down-staging and thus increased rates of breast-conserving surgery. Response to NACT is currently using 2 endpoints: clinical and pathological complete response (cCR and pCR). pCR, mostly defined as no histopathological residual invasive as well as non-invasive cancer in the breast or lymph nodes after NACT, correlates strongly with the long-term outcome in this BC subtype [23, 25-27]. Various prospective, multicenter, and randomized studies have investigated the combination of trastuzumab with chemotherapy in patients with HER2positive BC [28-30]. One of these studies, the GeparQuattro trial, a 3-arm study of the German Breast Group/Gynaecologic Oncology Study Group, assessed the effect of epirubicin (E) plus cyclophosphamide (C) followed by 1 of 3 different docetaxel-based regimens on pCR rates. In each arm, patients with HER2-positive BC received trastuzumab from the initiation of EC for 52 weeks. The pCR rate was $31.7 \%$ for patients with HER2-positive BC who received trastuzumab plus chemotherapy versus $15.7 \%$ for patients with HER2-negativ BC who received only chemotherapy ( $p$ $<0.001$ ). This study demonstrated that a higher $\mathrm{pCR}$ rate could be expected in candidates for NACT with HER2-positive BC treated concomitantly with trastuzumab and antracycline/taxane-based chemotherapy over 24-36 weeks [28]. With the development of other HER2-targeted drugs, the evaluation of a dual blockade in the treatment of $\mathrm{BC}$ has become attractive. The NeoALTTO (NeoAdjuvant Lapatinib and/or Trastuzumab Treatment Optimization) and the GeparQuinto study analyzed the combination of trastuzumab with lapatinib whereas the NeoSphere (Neoadjuvant Study of Pertuzumab and Herceptin in an Early Regimen Evaluation) study investigated the combination of trastuzumab and pertuzumab [31-33]. The 3-arm NeoALTTO study assessed the effect of paclitaxel plus lapatinib versus paclitaxel plus trastuzumab versus paclitaxel plus lapatinib plus trastuzumab on the $\mathrm{pCR}$ rate in women with operable HER2-positive BC. The pCR rate was significantly higher in the combination group (51.3\%) than in the trastuzumab group $(29.5 \%)$ or in the lapatinib group $(24.7 \%)$ proving that dual HER2 blockade is better than single anti-HER2 therapy. The NeoALTTO study recently reported at 3.8 years of follow-up, and there was no significant difference in event-free survival between the combination arm and the trastuzumab arm [34]. NeoSphere was a 4-arm-trial (arm A: trastuzumab plus docetaxel vs. arm B: pertuzumab plus trastuzumab plus docetaxel vs. arm C: pertuzumab plus trastuzumab vs. arm D: pertuzumab plus docetaxel). Significantly improved pCR rates were found in arm B with dual inhibition of HER2 and chemotherapy (45.8\%) compared to arm A with trastuzumab and docetaxel (29\%) or arm D with pertuzumab and docetaxel (24\%). Interestingly, arm C with only dual HER2-targeted therapy showed a low pCR rate (16.8\%). The number of serious adverse events was similar in arms A, B and D, but lower in arm C [32], so that toxicity seems to not be increased with use of a triplet regimen.

Furthermore, the TRYPHAENA study, a multicenter open-label phase II trial, evaluated the cardiac tolerability of a dual blockade with trastuzumab and pertuzumab combined with anthracyclinecontaining or -free standard chemotherapy in the neoadjuvant treatment of operable locally advanced or inflammatory HER2positive BC. 225 patients were randomized to 3 arms: A) FEC (5-fluorouracil, epirubicin, cyclophosphamide) for 3 cycles followed by docetaxel for 3 cycles, with trastuzumab and pertuzumab given concurrently throughout; B) FEC for 3 cycles followed by docetaxel with concurrent trastuzumab and pertuzumab for $3 \mathrm{cy}$ cles; and C) docetaxel plus carboplatin with concurrent trastuzumab and pertuzumab for 6 cycles. All patients then received trastuzumab to complete 1 year. Left ventricular systolic dysfunction (LVSD) was low across all arms (arm A: 5.6\%, arm B: 4.0\%, arm C: $2.6 \%)$. These data showed that neoadjuvant application of pertuzumab and trastuzumab, given concurrently or sequentially with an anthracycline-based chemotherapy or concurrently with a carboplatin-based chemotherapy, resulted in a low incidence of LVSD. However, cardiac function should be monitored in patients who receive adjuvant trastuzumab-based therapy. Moreover, assessment of pCR showed that all 3 therapies were highly effective, with pCR rates between 57.3 and 66.2\% [35].

\section{Adjuvant Therapy}

A 2008 meta-analysis including the adjuvant trastuzumab trials HERA, NCCTG-N9831, NSABP-B31, BIRCG-006, FinHER, and PACS-04 [36] could show a mean decrease in recurrence risk of about $37 \%$ and in mortality risk of about $34 \%$.

The 2 American trials, NCCTG-N9831 (North Central Cancer Treatment Group) and NSABP-B31 (National Surgical Adjuvant Breast and Bowel Project), had a similar design using paclitaxel either weekly or 3-weekly concurrently or sequentially with trastuzumab after 4 cycles of doxorubicin (A) and cyclophosphamide (C). The median follow-up was 8.4 years. An improvement in DFS was seen with a significant risk reduction in disease events of $40 \%$ 
as well as an improvement in OS with a significant risk reduction of $37 \%$ with the combination of trastuzumab and paclitaxel after 4 cycles AC 3-weekly [37].

The HERA (HERceptin Adjuvant) trial included 5,090 patients with HER2-positive BC after completion of the locoregional therapy and a variety of adjuvant chemotherapy regimens. Patients were randomized to 1 year or 2 years of therapy with trastuzumab, or to placebo. In contrast to NCCTG-N9831 and NSABP-B31, trastuzumab was given exclusively as sequential therapy. A first analysis of the study after 1 year of follow-up showed a significant reduction in recurrence of $46 \%$ independent of the preceding chemotherapy regimen, lymph node status, or hormone receptor status, as well as a significant reduction in mortality of $44 \%$ [38]. The final analysis after a median follow-up of 8 years could not show any benefit of a 2-year therapy with trastuzumab compared to 1-year standard therapy. Moreover, in the 2-year arm, the reported rate of cardiac events was higher than in the 1-year arm. The authors concluded that 1 year of treatment provided a significant DFS and OS benefit compared with observation and should remain the standard of care [39].

The efficacy of a shortened trastuzumab application was analyzed in the FinHER and the PHARE trials. Whereas the FinHER trial could demonstrate a benefit (hazard ratio (HR) for recurrence or death in the trastuzumab group compared to the control group $0.42 ; \mathrm{p}=0.01$ ) with a 9-week treatment regimen in a small cohort of patients $(n=232)$, the larger PHARE trial $(n=3,000)$ after 3.5 years of follow-up failed to show that 6 months of treatment with trastuzumab was non-inferior to 12 months of trastuzumab with regard to DFS (13 vs. 10.4\%, HR 1.28) as well as OS (HR 1.47) [40, 41].

The above adjuvant trials exclusively applied polychemotherapy backbones. However, another focus was reducing cytotoxic regimes as combination partners for trastuzumab. The APT trial was a single-group multicenter study evaluating single standard treatment and included 406 patients with tumors measuring up to $3 \mathrm{~cm}$ and lymph node-negative, HER2-positive BC. The patients received weekly treatment with paclitaxel and trastuzumab for 12 weeks, followed by 9 months of trastuzumab monotherapy. After a 4-year median follow-up, results showed a 3-year rate of survival free from invasive disease of about $98.7 \%$. In individual cases, monochemotherapy with weekly paclitaxel as an anthracycline-free combination partner for trastuzumab should be considered. Based on the risk/ benefit ratio, considerations should be made for anthracycline-free regimens in stage I disease [42]. Another adjuvant trial, BCIRG-006 (Breast Cancer International Research Group), analyzed the effectiveness and safety of nonanthracycline-based regimen and the efficacy of trastuzumab in order to reduce cardiac toxicity. 3,222 patients were randomized in this study and treated with doxorubicin, cyclophosphamide, and docetaxel (ACT), ACT plus trastuzumab for 1 year (AC-TH), or with an anthracycline-free regimen consisting of docetaxel, carboplatin, and 52 weeks of trastuzumab (TCH). This trial confirmed the importance of trastuzumab for the treatment of HER2-positive BC showing OS and DFS benefit for both trastuzumab-containing regimes compared to ACT. Moreover, no significant difference in OS and DFS could be demonstrated be- tween AC-TH (OS 92\%, DFS 84\%) and TCH (OS 91\%, DFS 81\%), but the TCH regimen showed lower cardiotoxicity. Especially for patients with high risk of heart failure, TCH seems to be an appropriate anthracycline-free alternative [43].

A further concern represents the dual inhibition of HER2 in adjuvant therapy. The ALTTO trial (Adjuvant Lapatinib and/or Trastuzumab Treatment Optimization) randomized 8,381 patients and was the largest study analyzing the efficacy of combination therapy in the adjuvant treatment in HER2-positive BC. Women were treated after surgery and chemotherapy (anthracycline-free vs. anthracycline-based regimens) with trastuzumab (T) alone, lapatinib (L) alone, trastuzumab followed by lapatinib $(\mathrm{T} \diamond \mathrm{L})$, or trastuzumab and lapatinib concurrently (TL). Because of an interim analysis suggesting inferiority, the lapatinib arm was prematurely closed. The 4-year DFS in the other arms, T, T $\diamond \mathrm{L}$ and TL, was 86, 87 and $88 \%$, respectively. Moreover, the combination of trastuzumab with lapatinib compared to trastuzumab alone did not show any benefit regarding OS, but adverse events were more likely to occur in patients treated with lapatinib [44].

\section{Metastatic Breast Cancer Therapy}

As in the adjuvant setting, treatment strategies in metastatic breast cancer (MBC) are based on tumor biology as well as clinical needs. Different parameters such as possible combination with targeted agents, previous treatments, aggressiveness of the disease, sites of metastasis, comorbidities, as well as patient preference and expectations guide the choice of treatment. In the case of MBC, monochemotherapy is preferred to polychemotherapy. One of the first studies comparing single-agent chemotherapy with or without trastuzumab was the trial by Slamon et al. [45]. Concurrent treatment with trastuzumab and first-line chemotherapy was associated with a significantly longer time to disease progression, higher response rates, longer duration of response, and improved OS. The CLEOPATRA study, a phase III trial, compared the efficacy and safety of pertuzumab, trastuzumab and docetaxel with placebo, trastuzumab and docetaxel in patients with HER2-positive firstline MBC. The dual-blockade arm showed significant benefit regarding both progression-free survival (PFS) and OS compared to the placebo arm (PFS: 12.4 vs. 18.7 months, OS: 56.5 vs. 40.8 months) [46].

Furthermore, T-DM1 is approved as a further HER2-targeted treatment in $\mathrm{MBC}$, especially for patients pretreated with trastuzumab and early recurrence of the disease. The EMILIA trial, a phase III trial, evaluated the efficacy and safety of T-DM1 compared with lapatinib plus capecitabine in patients with HER2-positive locally advanced $\mathrm{BC}$ or $\mathrm{MBC}$ following prior trastuzumabbased and taxane-containing chemotherapy [47]. Whereas PFS and median OS were significantly better for the T-DM1 arm (PFS: 9.6 vs. 6.4 months, $\mathrm{p}<0.001$; OS: 30.9 vs. 25.1 months, $\mathrm{p}<0.001$ ), the rate of toxicities was higher in the lapatinib plus capecitabine arm. In addition, the MARIANNE study, a phase III trial, randomized 1,095 patients with first-line HER2-positive MBC treated with 
T-DM1 plus placebo versus T-DM1 plus pertuzumab versus trastuzumab plus taxane-based chemotherapy (paclitaxel or docetaxel). No significant difference was shown regarding PFS in the 3 different arms [48]. Therefore, T-DM1 represents a recommended treatment alternative for patients with HER2-positive MBC in the first-line setting diagnosed less than 6 months after adjuvant therapy using taxane and trastuzumab or in the second-line treatment after trastuzumab therapy.

\section{Summary}

In the case of HER2-positive $\mathrm{BC}$ or $\mathrm{MBC}$, targeted therapies are well established and make selective treatment possible. Based on the above studies, the breast committee of the AGO (Arbeitsgemeinschaft Gynäkologische Onkologie or German Gynaecological Oncology Group) preferred NACT as a therapeutic option in patients who have a clear indication for adjuvant postoperative chemotherapy (LoE $1 / \mathrm{B} / \mathrm{AGO}+$ ), especially in patient subgroups where a pCR is associated with improved survival such as HER2positive $\mathrm{BC}(\mathrm{LoE} 1 \mathrm{a} / \mathrm{A} / \mathrm{AGO}++)$. Moreover, anti-HER2 treatment with trastuzumab is recommended to be given concurrently with taxane $(\mathrm{LoE} 1 \mathrm{a} / \mathrm{A} / \mathrm{AGO}++)$ for an optimal duration of 1 year $(\mathrm{LoE}$ 1b/A/AGO ++) as a standard treatment for early HER2-positive BC. Trastuzumab should be administered in all HER2- and nodalpositive $\mathrm{BC}$ as well as nodal-negative tumors $>1 \mathrm{~cm}$ with an indication for chemotherapy (LoE 1a/A/AGO ++). For smaller tumors between 0.5 and $1 \mathrm{~cm}$, an individual treatment decision based on additional risk factors has to be made. With regard to alternative anti-HER2 treatments, neither lapatinib nor dual therapy with lapatinib plus trastuzumab is recommended ( $\mathrm{LoE} 1 \mathrm{~b} / \mathrm{B} / \mathrm{AGO}-$ ). In the case of HER2-positive MBC, the combination of docetaxel, trastuzumab, and pertuzumab as first-line therapy is the standard of care (LoE 1b/A/AGO ++). As second-line therapy in MBC after treatment with trastuzumab, T-DM1 is the treatment of choice (LoE 1b/A/ AGO ++).

\section{Perspective}

Further trials, such as the ADAPT study a prospective multicenter randomized study, are trying to identify additional surrogate markers for therapy response and clinical outcome in the neoadjuvant setting [49]. Recruitment for ADAPT in the subgroup of HER2- and hormone receptor-positive BC (triple-positive BC) is already finalized. This substudy, in a 3-armdesign, is comparing T-DM1 alone versus T-DM1 plus endocrine therapy versus trastuzumab plus endocrine therapy. A first analysis with 130 patients was presented at the ASCO congress in June 2015. The pCR rates in the different arms were $40.5,45.8$, and $6.7 \%$, respectively. This data shows the strong effect of T-DM1 alone versus trastuzumab without chemotherapy. However, the additional effect of endocrine therapy is still being investigated. This information opens new perspectives for neoadjuvant therapy concepts for triple-positive $\mathrm{BC}$ with a possible de-escalation of classical chemotherapy in favor of targeted therapy. However, the effect on clinical outcome (OS and DFS) is not clear, and further results are to be awaited to confirm these first data.

\section{Disclosure Statement}

Both authors have no financial interest or affiliation with 1 or more organizations that could be perceived as a real or apparent conflict of interest in the context of the subject of this review.

\section{References}

1 Slamon DJ, Clark GM, Wong SG, et al.: Human breast cancer: correlation of relapse and survival with amplification of the HER-2/neu oncogene. Science 1987;235: 177-182.

2 Menard S, Tagliabue E, Campiglio M, Pupa SM: Role of HER2 gene overexpression in breast carcinoma. J Cell Physiol 2000;182:150-162.

3 Sjogren S, Inganäs M, Lindgren A, et al.: Prognostic and predictive value of c-erbB-2 overexpression in primary breast cancer, alone and in combination with other prognostic markers. J Clin Oncol 1998;16:462-469.

4 Gabos Z, Sinha R, Hanson J, et al.: Prognostic significance of human epidermal growth factor receptor positivity for the development of brain metastasis after newly diagnosed breast cancer. J Clin Oncol 2006;24: 5658-5663.

5 Dawood S, Broglio K, Buzdar AU, et al.: Prognosis of women with metastatic breast cancer by HER2 status and trastuzumab treatment: an institutional-based review. J Clin Oncol 2010;28:92-98.

6 Graus-Porta D, Beerli RR, Daly JM, Hynes NE: ErbB-2, the preferred heterodimerization partner of all ErbB receptors, is a mediator of lateral signaling. EMBO J 1997; 16:1647-1655.
Olayioye MA: Update on HER-2 as a target for cancer therapy: intracellular signaling pathways of ErbB2/ HER-2 and family members. Breast Cancer Res 2001;3: 385-389.

8 Brennan PJ, Kumagai T, Berezov A, et al.: HER2/neu: mechanisms of dimerization/oligomerization. Oncogene 2000;19:6093-6101.

9 Luque-Cabal M, García-Teijido P, Fernández-Pérez Y, et al.: Mechanisms behind the resistance to trastuzumab in HER2-amplified breast cancer and strategies to overcome it. Clin Med Insights Oncol 2016;10(suppl 1): 21-30.

10 Baselga J, Albanell J, Molina MA, Arribas J: Mechanism of action of trastuzumab and scientific update. Semin Oncol 2001;28(5 suppl 16):4-11

11 Viani GA, Afonso SL, Stefano EJ, et al.: Adjuvant trastuzumab in the treatment of her-2-positive early breast cancer: a meta-analysis of published randomized trials. BMC Cancer 2007;7:153.

12 Barthelemy P, Leblanc J, Goldbarg V, et al.: Pertuzumab: development beyond breast cancer. Anticancer Res 2014;34:1483-1491.
Franklin MC, Carey KD, Vajdos FF, et al.: Insights into ErbB signaling from the structure of the ErbB2-pertuzumab complex. Cancer Cell 2004;5:317-328.

14 Nahta R, Hung MC, Esteva FJ: The HER-2-targeting antibodies trastuzumab and pertuzumab synergistically inhibit the survival of breast cancer cells. Cancer Res 2004;64:2343-2346.

15 LoRusso PM, Weiss D, Guardino E, et al.: Trastuzumab emtansine: a unique antibody-drug conjugate in development for human epidermal growth factor receptor 2-positive cancer. Clin Cancer Res 2011;17: 6437-6447.

16 Lewis Phillips GD, Li G, Dugger DL, et al.: Targeting HER2-positive breast cancer with trastuzumab-DM1, an antibody-cytotoxic drug conjugate. Cancer Res 2008;68:9280-9290.

17 Dzimitrowicz H, Berger M, Vargo C, et al.: T-DM1 activity in metastatic human epidermal growth factor receptor 2-positive breast cancers that received prior therapy with trastuzumab and pertuzumab. J Clin Oncol 2016;Epub ahead of print.

18 Bourdeanu L, Luu T: Targeted therapies in breast cancer: implications for advanced oncology practice. J Adv Pract Oncol 2014;5:246-260. 
19 Konecny GE, Pegram MD, Venkatesan N, et al.: Activity of the dual kinase inhibitor lapatinib (GW572016) against HER-2-overexpressing and trastuzumab-treated breast cancer cells. Cancer Res 2006;66:1630-1639.

20 Spector NL, Xia W, Burris H 3rd, et al.: Study of the biologic effects of lapatinib, a reversible inhibitor of ErbB1 and ErbB2 tyrosine kinases, on tumor growth and survival pathways in patients with advanced malignancies. J Clin Oncol 2005;23:2502-2512.

21 Nelson MH, Dolder CR: Lapatinib: a novel dual tyrosine kinase inhibitor with activity in solid tumors. Ann Pharmacother 2006;40:261-269.

22 Burris HA 3rd: Dual kinase inhibition in the treatment of breast cancer: initial experience with the EGFR/ ErbB-2 inhibitor lapatinib. Oncologist 2004;(9 suppl 3): $10-15$.

23 Fisher B, Bryant J, Wolmark N, et al.: Effect of preoperative chemotherapy on the outcome of women with operable breast cancer. J Clin Oncol 1998;16:2672-2685.

24 Mauri D, Pavlidis N, Ioannidis J: Neoadjuvant versus adjuvant systemic treatment in breast cancer: a metaanalysis. J Natl Cancer Inst 2005;97:188-194.

25 Buzdar AU, Ibrahim NK, Francis D, et al.: Significantly higher pathologic complete remission rate after neoadjuvant therapy with trastuzumab, paclitaxel, and epirubicin chemotherapy: results of a randomized trial in human epidermal growth factor receptor 2-positive operable breast cancer. J Clin Oncol 2005;23:36763685.

26 Cleator SJ, Makris A, Ashley SE, et al.: Good clinical response of breast cancers to neoadjuvant chemoendocrine therapy is associated with improved overall survival. Ann Oncol 2005; 16:267-272.

27 Symmans WF, Peintinger F, Hatzis C, et al.: Measurement of residual breast cancer burden to predict survival after neoadjuvant chemotherapy. J Clin Oncol 2007;25:4414-4422.

28 Untch M, Rezai M, Loibl S, et al.: Neoadjuvant treatment with trastuzumab in HER2-positive breast cancer: results from the GeparQuattro study. J Clin Oncol 2010;28:2024-2031.

29 Pierga JY, Delaloge S, Espié M, et al.: A multicenter randomized phase II study of sequential epirubicin/ cyclophosphamide followed by docetaxel with or without celecoxib or trastuzumab according to HER2 status, as primary chemotherapy for localized invasive breast cancer patients. Breast Cancer Res Treat 2010; 122:429-437.

30 Chang HR: Trastuzumab-based neoadjuvant therapy in patients with HER2-positive breast cancer. Cancer 2010;116:2856-2867.
31 Baselga J, Bradbury I, Eidtmann H, et al.: Lapatinib with trastuzumab for HER2-positive early breast can cer (NeoALTTO): a randomised, open-label, multicentre, phase 3 trial. Lancet 2012;379:633-640.

32 Gianni L, Pienkowski T, Im YH, et al.: Efficacy and safety of neoadjuvant pertuzumab and trastuzumab in women with locally advanced, inflammatory, or early HER2-positive breast cancer (NeoSphere): a randomised multicentre, open-label, phase 2 trial. Lancet Oncol 2012;13:25-32.

33 Untch M, Loibl S, Bischoff J, et al.: Lapatinib versus trastuzumab in combination with neoadjuvant anthracycline-taxane-based chemotherapy (GeparQuinto, GBG 44): a randomised phase 3 trial. Lancet Oncol 2012;13:135-144.

34 De Azambuja E, Holmes AP, Piccart-Gebhart M, et al.: Lapatinib with trastuzumab for HER2-positive early breast cancer (NeoALTTO): survival outcomes of a randomised, open-label, multicentre, phase 3 trial and their association with pathological complete response. Lancet Oncol 2014;15:1137-1146.

35 Schneeweiss A, Chia S, Hickish T, et al.: Pertuzumab plus trastuzumab in combination with standard neoadjuvant anthracycline-containing and anthracyclinefree chemotherapy regimens in patients with HER2positive early breast cancer: a randomized phase II cardiac safety study (TRYPHAENA). Ann Oncol 2013; 24:2278-2284.

36 Dahabreh IJ, Linardou H, Siannis F, et al.: Trastuzumab in the adjuvant treatment of early-stage breast cancer: a systematic review and meta-analysis of randomized controlled trials. Oncologist 2008; 13:620-630.

37 Perez EA, Perez EA, Romond EH, Suman VJ, et al.: Trastuzumab plus adjuvant chemotherapy for human epidermal growth factor receptor 2-positive breast cancer: planned joint analysis of overall survival from NSABP B-31 and NCCTG N9831. J Clin Oncol 2014; 32:3744-3752.

38 Smith I, Procter M, Gelber RD, et al.: 2-year follow-up of trastuzumab after adjuvant chemotherapy in HER2positive breast cancer: a randomised controlled trial. Lancet 2007;369:29-36.

39 Goldhirsch A, Gelber RD, Piccart-Gebhart MJ, et al.: 2 years versus 1 year of adjuvant trastuzumab for HER2positive breast cancer (HERA): an open-label, randomised controlled trial. Lancet 2013;382:1021-1028.

40 Joensuu H, Kellokumpu-Lehtinen PL, Bono P, et al.: Adjuvant docetaxel or vinorelbine with or without trastuzumab for breast cancer. N Engl J Med 2006;354: 809-820.
41 Pivot X, Romieu G, Debled M, et al.: 6 months versus 12 months of adjuvant trastuzumab for patients with HER2-positive early breast cancer (PHARE): a randomised phase 3 trial. Lancet Oncol 2013;14:741-748.

42 Tolaney SM, Barry WT, Dang CT, et al.: Adjuvant paclitaxel and trastuzumab for node-negative, HER2positive breast cancer. N Engl J Med 2015;372:134-141.

43 Slamon D, Eiermann W, Robert N, et al.: Adjuvant trastuzumab in HER2-positive breast cancer. N Engl J Med 2011;365:1273-1283.

44 Piccart-Gebhart M, Holmes AP, Baselga J, et al.: First results from the phase III ALTTO trial (BIG 02-06; NCCTG 063D) comparing one year of anti-HER2 therapy with lapatinib alone (L), trastuzumab alone (T), their sequence $(T \rightarrow L)$ or their combination $(L+T)$ in the adjuvant treatment of HER2-positive early breast cancer (EBC). J Clin Oncol 2014;32(suppl):abstr LBA4.

45 Slamon DJ, Leyland-Jones B, Shak S, et al.: Use of chemotherapy plus a monoclonal antibody against HER2 for metastatic breast cancer that overexpresses HER2. N Engl J Med 2001;344:783-792.

46 Swain SM, Kim SB, Cortés J, et al.: Pertuzumab, trastuzumab, and docetaxel for HER2-positive metastatic breast cancer (CLEOPATRA study): overall survival results from a randomised, double-blind, placebo-controlled, phase 3 study. Lancet Oncol 2013;14:461-471.

47 Verma S, Dieras V, Gianni L, et al.: EMILIA: a phase III, randomized, multicenter study of trastuzumab-DM1 (T-DM1) compared with lapatinib (L) plus capecitabine (X) in patients with HER2-positive locally advanced $\mathrm{MBC}$ and previously treated with a trastuzumab-based regimen. J Clin Oncol 2011;29:15s(suppl):abstr TPS116.

48 Ellis PA, Barrios CH, Eiermann W, et al.: Phase III, randomized study of trastuzumab emtansine (T-DM1) +- pertuzumab $(\mathrm{P})$ vs trastuzumab + taxane $(\mathrm{HT})$ for first-line treatment of HER2-positive MBC: Primary results from the MARIANNE study. J Clin Oncol 2015; 33(suppl):abstr 507.

49 Hofmann D, Nitz U, Gluz O, et al.: WSG ADAPT adjuvant dynamic marker-adjusted personalized therapy trial optimizing risk assessment and therapy response prediction in early breast cancer: study protocol for a prospective, multi-center, controlled, nonblinded, randomized, investigator initiated phase II/III trial. Trials 2013;14:261. 